\title{
Right- and left-sided stage III colon cancers present different prognostic outcomes of oxaliplatin-based adjuvant chemotherapy after curative resection
}

This article was published in the following Dove Press journal:

Cancer Management and Research

Jianhong Peng, ',* Cong Li, ${ }^{1, *}$ Fulong Wang,' Huizhong Zhang, ${ }^{2}$ Weiwei Xiao, ${ }^{3} \mathrm{Hui} \mathrm{Li},{ }^{4}$ Zhenhai Lu,' Zhizhong Pan,' Xiaojun Wu,' Rongxin Zhang'

'Department of Colorectal Surgery, Sun Yat-sen University Cancer Center, State Key Laboratory of Oncology in South China, Collaborative Innovation Center for Cancer Medicine, Guangzhou 510060 , People's Republic of China; ${ }^{2}$ Department of Pathology, Sun Yat-sen University Cancer Center, State Key Laboratory of Oncology in South China, Collaborative Innovation Center for Cancer Medicine, Guangzhou 510060 , People's Republic of China; ${ }^{3}$ Department of Radiation Oncology, Sun Yat-sen University Cancer Center, State Key Laboratory of Oncology in South China, Collaborative Innovation Center for Cancer Medicine, Guangzhou 510060, People's Republic of China; ${ }^{4}$ Department of Gynecological Oncology, Sun Yatsen Memorial Hospital, Sun Yat-sen University, Guangzhou 510060, People's Republic of China

*These authors contributed equally to this work

Correspondence: Xiaojun Wu; Rongxin Zhang Department of Colorectal Surgery, Sun Yat-sen University Cancer Center, State Key Laboratory of Oncology in South China, Collaborative Innovation Center for Cancer Medicine, 65I Dongfeng Road East, Guangzhou 510060, People's Republic of China

Tel +86 2087343124

$\mathrm{Fax}+862087343637$

Email zhangrx@sysucc.org.cn ; wuxj@sysucc. org.cn
Background: Growing evidence has suggested that right-sided colon cancer (RCC) and leftsided colon cancer (LCC) should be considered as different tumor entities. However, stage III colon cancer is currently treated as the same entity with uniform therapy. This study was aimed at investigating the prognostic influence of tumor location in patients with stage III colon cancer receiving adjuvant chemotherapy after curative resection.

Patients and methods: We retrospectively analyzed 274 eligible patients with stage III colon cancer undergoing curative tumor resection followed by adjuvant chemotherapy with oxaliplatin and capecitabine between December 2007 and December 2013. Disease-free survival (DFS) and overall survival (OS) were analyzed using Kaplan-Meier and log-rank tests, and prognostic factors were identified by Cox regression methods.

Results: Patients with RCC exhibited lower hemoglobin levels ( $23.6 \%$ vs. 9.8\%; $P=0.002)$, larger tumor size $(60.6 \%$ vs. $40.9 \% ; P=0.001)$, and a higher proportion of 12 or more resected lymph nodes $(86.4 \%$ vs. $64.6 \% ; P<0.001)$ than patients with LCC. Grade 1 neurotoxicity was more common in patients with RCC than in those with LCC (53.6\% vs. $40.9 \%$; $P=0.037)$. RCC was significantly associated with a shorter 3-year OS than LCC, whereas a difference was noted only for stage IIIC and not stage IIIA or stage IIIB colon cancer. Multivariate analyses revealed that RCC was independently associated with a worse 3-year OS (hazard ratio: 2.213 ; $95 \% \mathrm{CI}$ : $1.063-4.606 ; P=0.002$ ). In addition, an increase in 3-year OS and DFS after 6-8 cycles of adjuvant chemotherapy was only observed in patients with RCC and not in those with LCC.

Conclusion: This study indicated that RCC has a worse prognostic outcome for stage III colon cancer, and a full course of adjuvant chemotherapy should be suggested for patients with RCC. Keywords: left-sided colon cancer, right-sided colon cancer, stage III, adjuvant chemotherapy, outcome, colon cancer, location, resection, prognosis

\section{Introduction}

Curative surgical resection followed by adjuvant chemotherapy has been universally proposed as a standard clinical practice for patients with resected stage III colon cancer. ${ }^{1,2}$ It is well established that adjuvant therapy with a combined regimen of oxaliplatin and fluoropyrimidines (5-fluorouracil [5-FU]) is a more effective treatment, and it was shown to increase the disease-free survival (DFS) rate for stage III colon cancer from $4.4 \%$ to $12.3 \%$ compared with 5-FU alone. ${ }^{3-5}$ Additionally, administration of a fixed 6-month regimen of oxaliplatin-containing adjuvant chemotherapy is generally recommended., ${ }^{1,6}$ 
In fact, approximately $30 \%$ of patients failed to complete the full planned course of adjuvant chemotherapy after surgical resection because of therapeutic toxicity, poor tolerance of intensive therapies, and economic burden. ${ }^{3,5,7}$ Moreover, $25 \%-30 \%$ of patients with stage III colon cancer ultimately develop distant metastases despite receiving curative treatment. ${ }^{8,9}$ Therefore, the management of stage III colon cancer remains challenging, and understanding the prognostic factors affecting stage III colon cancer is important to individualize adjuvant chemotherapy according to patient classification.

Currently, colon cancer is not recognized as a single entity but is divided into proximal (right) or distal (left) compartments at the splenic flexure. ${ }^{10,11}$ Accumulating evidence has revealed that cancers arising from different sides of the colon exhibit different features, such as epidemiological incidence, physiological characteristics, molecular alterations, and even survival outcome. ${ }^{12-15}$ Compared with left-sided colon cancer (LCC), right-sided colon cancer (RCC) more commonly presents as poorly differentiated and locally advanced tumors with specific molecular characteristics that include a highlevel 5'-C-phosphate- $-\mathrm{G}-3$ ' $(\mathrm{CpG})$ island methylator phenotype (CIMP), microsatellite instability (MSI), and BRAF mutations as well as a worse survival outcome. ${ }^{13,14,16}$ However, the survival benefit of oxaliplatin-containing adjuvant chemotherapy after curative resection for rightand left-sided stage III colon cancers remains limited. ${ }^{17,18}$ In addition, it remains unknown whether primary tumor location is associated with the acute toxicities that prevented the completion of adjuvant chemotherapy. Moreover, based on the different abovementioned features, the survival benefit of the full course of adjuvant chemotherapy for site-specific stage III colon cancers might also be questioned. We hypothesized that the efficacy of oxaliplatin-containing adjuvant chemotherapy is not only influenced by tumor stage but also by tumor location.

To test this hypothesis, this study explored the association of site-specific colon cancers with survival outcome and chemotherapy-related toxicities. In addition, we also aimed to identify subgroups of patients with specific-sided colon cancer who benefited from a full course of adjuvant chemotherapy to optimize the duration of adjuvant chemotherapy for stage III colon cancer.

\section{Patients and methods}

\section{Patient selection}

This study included 321 consecutive patients diagnosed with stage III colon cancer who received tumor resection followed by adjuvant chemotherapy from December 2007 to December 2013 at Sun Yat-sen University Cancer Center. The enrolled patients met the following inclusion criteria: 1) histologically confirmed adenocarcinoma; 2) American Society of Anesthesiologists class I-II; 3) curative resection for colon tumor; 4) XELOX adjuvant chemotherapy (oxaliplatin $130 \mathrm{mg} / \mathrm{m}^{2}$ administered intravenously on day 1 and capecitabine $1000 \mathrm{mg} / \mathrm{m}^{2}$ administered orally twice daily on days 1-14 for a 3-week cycle); 5) no anti-cancer therapy before surgery; and 6) postoperative follow-up period $\geq 3$ months. Forty-seven patients were excluded for the following reasons: postoperative chemotherapy with other regimens ( $\mathrm{n}$ $=36)$; lost to follow-up $(\mathrm{n}=7)$; and palliative tumor resection $(n=6)$. In total, 274 eligible patients with stage III colon cancer were attentively reviewed from the medical records, including patient demographics, tumor characteristics, and adjuvant chemotherapy toxicity. This study was performed according to the ethical standards of the World Medical Association Declaration of Helsinki and was approved by the institutional review board and independent ethics committees of Sun Yat-sen University Cancer Center. Informed consent was waived off because of the nature of retrospective study, and confidentiality was maintained for patient data.

\section{Parameter definitions}

The pathological tumor-node-metastasis (TNM) classification was defined according to the criteria of the 2010 American Joint Commission on Cancer (AJCC)/International Union Against Cancer. Tumor location was confirmed by intraoperation. Based on the definition in previous studies, ${ }^{19,20}$ RCC included the cecum, ascending colon, hepatic flexure, and transverse colon, whereas LCC included the splenic flexure, descending colon, and sigmoid colon. Major toxicities during adjuvant chemotherapy were graded according to the National Cancer Institute Common Toxicity Criteria version 4.0.

\section{Follow-up}

The follow-up data for all of the included patients were available from the follow-up system for analysis. Routine check-up, blood tests to detect carcinoembryonic antigen (CEA) and carbohydrateantigen19-9 (CA19-9), abdominal ultrasonography, and chest radiography were conducted every 3 months. Chest/abdominal/pelvic CT scans and colonoscopies were performed annually. The last follow-up visit was in December 2016. Overall survival (OS) was defined as the interval (in months) from the date of surgery until death from any cause or the last follow-up, whereas DFS was defined as the interval (in months) from tumor resection to disease recurrence, death, or the last follow-up. 


\section{Statistical analysis}

All of the data were analyzed using IBM SPSS statistics software, version 21.0 (IBM Corp., Armonk, NY, USA). The correlations between clinicopathological variables and tumor location (RCC vs. LCC) were assessed using chisquared test or Fisher's exact test. The 3-year DFS and OS rates were calculated with the Kaplan-Meier method, and the differences were compared with the log-rank test. The Cox proportional hazards model was used for univariate and multivariate analyses. Variables that were statistically significant in univariate Cox models were further assessed with multivariate Cox models using a forward stepwise method. Hazard ratios (HRs) and 95\% CIs were subsequently calculated. All of the statistical tests used in this study were two-sided, and a $P$-value $<0.05$ was considered significant.

\section{Results \\ Patient characteristics}

The clinicopathological and treatment data regarding all eligible patients are summarized in Table 1. Of the 274 patients,

Table I Clinicopathological characteristics of patients with stage III colon cancer

\begin{tabular}{|c|c|c|c|c|}
\hline Variables & Overall cases (\%) & Right-sided colon (n, \%) & Left-sided colon $(n, \%)$ & $P$-value \\
\hline Total & 274 & $110(40.1)$ & $164(59.9)$ & \\
\hline Age (years) & & & & 0.974 \\
\hline$\leq 60$ & $189(69.0)$ & $76(69.1)$ & $113(68.9)$ & \\
\hline$>60$ & $85(31.0)$ & $34(20.9)$ & $5 I(3 I . I)$ & \\
\hline Gender & & & & 0.401 \\
\hline Male & $156(56.9)$ & $66(60.0)$ & $90(54.9)$ & \\
\hline Female & $118(43.1)$ & $44(40.0)$ & $74(45.1)$ & \\
\hline Baseline BMI $\left(\mathrm{kg} / \mathrm{m}^{2}\right)$ & & & & 0.992 \\
\hline$<18.5$ & $28(10.2)$ & II (I0.0) & $17(10.4)$ & \\
\hline $18.5-25.0$ & $187(68.2)$ & $75(68.2)$ & $111(68.1)$ & \\
\hline$>25$ & $59(21.5)$ & $24(21.8)$ & $35(21.5)$ & \\
\hline Baseline hemoglobin $(g / L)$ & & & & 0.002 \\
\hline$<90$ & $42(15.3)$ & $26(23.6)$ & $16(9.8)$ & \\
\hline$\geq 90$ & $232(84.7)$ & $84(76.4)$ & $148(90.2)$ & \\
\hline \multicolumn{5}{|l|}{ Tumor size $(\mathrm{cm})$} \\
\hline$\leq 4$ & I $40(5 \mathrm{I} . \mathrm{l})$ & $43(39.1)$ & $97(59.1)$ & 0.001 \\
\hline$>4$ & 134 (48.9) & $67(60.9)$ & $67(40.9)$ & \\
\hline \multicolumn{5}{|l|}{ Differentiation } \\
\hline Well/moderate & $207(75.5)$ & $77(70.0)$ & $130(79.3)$ & 0.080 \\
\hline Poor/undifferentiated & $67(24.5)$ & $33(30.0)$ & $34(20.7)$ & \\
\hline \multicolumn{5}{|l|}{ T stage } \\
\hline $\mathrm{TI}-\mathrm{T} 2$ & $12(4.4)$ & $2(1.8)$ & $10(6.1)$ & 0.138 \\
\hline T3 & $121(44.2)$ & $54(49.1)$ & $67(40.9)$ & \\
\hline T4 & $|4|(5 \mid .5)$ & $54(49.1)$ & $87(53.0)$ & \\
\hline Number of resected lymph nodes & & & & $<0.001$ \\
\hline$<12$ & $73(26.6)$ & $15(13.6)$ & $58(35.4)$ & \\
\hline$\geq 12$ & $201(73.4)$ & $95(86.4)$ & $106(64.6)$ & \\
\hline \multicolumn{5}{|l|}{$N$ stage } \\
\hline $\mathrm{NI}$ & $189(69.0)$ & $77(70.0)$ & $112(68.3)$ & 0.765 \\
\hline N2 & $85(31.0)$ & $33(30.0)$ & $52(31.7)$ & \\
\hline \multicolumn{5}{|l|}{ TNM stage } \\
\hline IIIA & $10(3.6)$ & $2(1.8)$ & $8(4.9)$ & 0.409 \\
\hline IIIB & $202(73.7)$ & $82(74.5)$ & $120(73.2)$ & \\
\hline IIIC & $62(22.6)$ & $26(23.6)$ & $36(22.0)$ & \\
\hline Preoperative serum CEA (ng/mL) & & & & 0.771 \\
\hline$\leq 5$ & $159(58.0)$ & $65(59.1)$ & $94(57.3)$ & \\
\hline$>5$ & $115(42.0)$ & 45 (40.9) & $70(42.7)$ & \\
\hline Cycles of adjuvant XELOX chemotherapy* & & & & 0.074 \\
\hline$<6$ & $54(19.5)$ & $27(24.5)$ & $26(15.9)$ & \\
\hline $6-8$ & $223(80.5)$ & $83(75.5)$ & $138(84.1)$ & \\
\hline
\end{tabular}

Notes: *XELOX adjuvant chemotherapy was administered as follows: oxaliplatin at a dose of $130 \mathrm{mg} / \mathrm{m}^{2}$ was administered intravenously on day I, and capecitabine was administered orally at a dosage of $1000 \mathrm{mg} / \mathrm{m}^{2}$ twice daily on days $\mathrm{I}-14$ for a 3-week cycle.

Abbreviations: BMI, body mass index; TNM, tumor-node-metastasis; CEA, carcinoembryonic antigen. 
$156(56.9 \%)$ were males and $118(43.1 \%)$ were female, with a median age of 55 years (range, 22-85 years). Regarding tumor location, 110 (40.1\%) patients had RCC, including $12(4.4 \%)$ cecal cancers, $47(17.2 \%)$ ascending colon cancers, $25(9.1 \%)$ hepatic flexure colon cancers, and $26(9.5 \%)$ transverse colon cancers, whereas 164 (59.9\%) patients had LCC, including $5(1.8 \%)$ splenic flexure colon cancers, 24 (8.8\%) descending colon cancers, and 135 (49.3\%) sigmoid colon cancers. The median number of resected lymph nodes and metastatic lymph nodes were 16 (range, 2-63) and 2 (range, 1-23), respectively. Regarding AJCC stage, 10 (3.6\%) patients were diagnosed with stage IIIA colon cancer, 202 (73.7\%) with stage IIIB colon cancer, and $62(22.6 \%)$ with stage IIIC colon cancer. Totally, $5(1.8 \%)$ patients experienced post-operative complications, including $3(1.1 \%)$ intestinal obstruction, 1 $(0.4 \%)$ anastomotic leakage, and $1(0.4 \%)$ incision infection. As shown in Figure 1, the median cycle of XELOX adjuvant chemotherapy for all of the patients was 6 (range, 1-8).

\section{Relationships between site-specific colon cancers, clinicopathological variables, and toxicities}

Relationships between site-specific colon cancers and clinical parameters are presented in Table 1. A lower baseline hemoglobin proportion $(<90 \mathrm{~g} / \mathrm{L})$ was more common in patients with RCC than in those with LCC $(23.6 \%$ vs. $9.8 \% ; P=0.002)$. Patients with RCC were more likely to exhibit a large tumor $(>4 \mathrm{~cm})$ than those with LCC $(60.6 \%$ vs. $40.9 \% ; P=0.001)$. A larger number of resected lymph nodes $(>12)$ occurred more frequently in RCC patients than in LCC patients $(86.4 \%$ vs. $64.6 \% ; P<0.001)$. However, there were no significant differences observed regarding

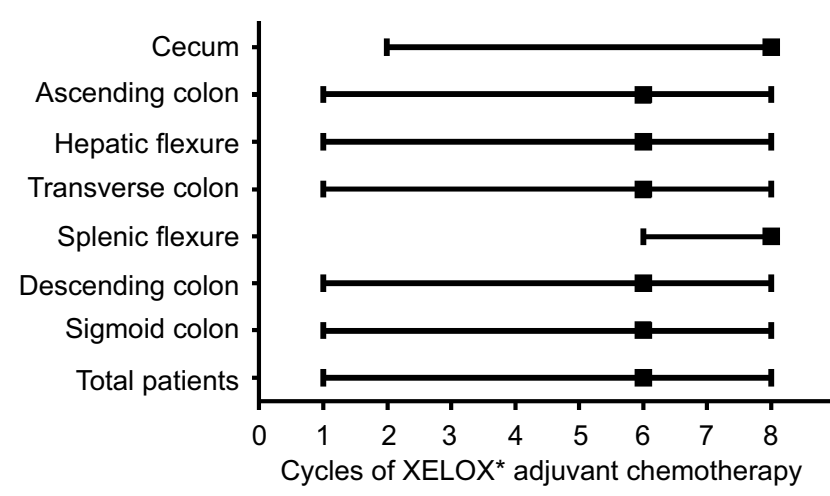

Figure I Median cycle of XELOX adjuvant chemotherapy received by patients for each tumor location.

Notes: *XELOX adjuvant chemotherapy was administered as follows: oxaliplatin at a dose of $130 \mathrm{mg} / \mathrm{m}^{2}$ was administered intravenously on day I, and capecitabine was administered orally at a dosage of $1000 \mathrm{mg} / \mathrm{m}^{2}$ twice daily on days $1-14$ for a 3-week cycle. age, gender, baseline body mass index, tumor differentiation, TNM stage, preoperative serum CEA level, and cycles of adjuvant chemotherapy.

The major toxicities during adjuvant chemotherapy are presented in Table 2 . Grade 1 neurotoxicity was more common in patients with RCC than in those with LCC (53.6\% vs. $40.9 \%, P=0.037)$. No significant differences were identified for leucopenia, thrombocytopenia, nausea and vomiting, diarrhea, hand-foot syndrome, or hepatic disorder.

\section{Survival analysis of site-specific colon cancers}

In this study, the median follow-up period for total patients was 46 months (range, 3-74 months). During this period, $53(19.3 \%)$ patients experienced tumor metastasis; in total, $30(10.9 \%)$ patients died of tumor progression. As shown in Table 3, abdominopelvic metastasis was more common in RCC patients than in LCC patients $(10.9 \%$ vs. $2.4 \%, P=0.003)$. The total postoperative metastasis, liver

Table 2 Treatment-related toxicities during adjuvant chemotherapy in patients with stage III colon cancer after curative resection

\begin{tabular}{|c|c|c|c|}
\hline Toxicities & $\begin{array}{l}\text { Right-sided } \\
\text { colon cancer } \\
(\mathrm{n}=1 \mathrm{I} 0, \%)\end{array}$ & $\begin{array}{l}\text { Left-sided } \\
\text { colon cancer } \\
(n=164, \%)\end{array}$ & $P$-value \\
\hline \multicolumn{4}{|l|}{ Leucopenia } \\
\hline Total & $63(57.3)$ & $93(56.7)$ & 0.926 \\
\hline Grade I-2 & $54(49.1)$ & $81(49.4)$ & 0.961 \\
\hline Grade 3-4 & $9(8.2)$ & $12(7.3)$ & 0.792 \\
\hline \multicolumn{4}{|c|}{ Thrombocytopenia } \\
\hline Total & $44(40.0)$ & $68(41.5)$ & 0.809 \\
\hline Grade I-2 & $32(29.1)$ & $54(32.9)$ & 0.502 \\
\hline Grade 3-4 & $12(10.9)$ & $14(8.5)$ & 0.511 \\
\hline \multicolumn{4}{|c|}{ Nausea/vomiting } \\
\hline Total & $22(20.0)$ & $36(22.0)$ & 0.698 \\
\hline Grade I & II (I0.0) & $21(12.8)$ & 0.734 \\
\hline Grade 2-3 & II (I0.0) & $15(9.1)$ & 0.813 \\
\hline \multicolumn{4}{|l|}{ Diarrhea } \\
\hline Total & $18(16.4)$ & $18(11.0)$ & 0.196 \\
\hline Grade I & $9(8.2)$ & $12(7.3)$ & 0.792 \\
\hline Grade 2-3 & $9(8.2)$ & $6(3.7)$ & 0.107 \\
\hline \multicolumn{4}{|c|}{ Hand-foot syndrome } \\
\hline Total & $57(51.8)$ & $68(4 \mid .5)$ & 0.092 \\
\hline Grade I & $47(42.7)$ & $53(32.3)$ & 0.079 \\
\hline Grade 2-3 & $10(9.1)$ & $15(9.1)$ & 0.988 \\
\hline \multicolumn{4}{|c|}{ Hepatic disorder } \\
\hline Total & $39(35.5)$ & $50(30.5)$ & 0.389 \\
\hline Grade I & $29(26.4)$ & $35(21.3)$ & 0.335 \\
\hline Grade 2-3 & $10(9.1)$ & $15(9.1)$ & 0.988 \\
\hline \multicolumn{4}{|c|}{ Neurotoxicity } \\
\hline Total & 78 (70.9) & $105(64.0)$ & 0.236 \\
\hline Grade I & $59(53.6)$ & $67(40.9)$ & 0.037 \\
\hline Grade 2-3 & $20(18.2)$ & $38(23.2)$ & 0.322 \\
\hline
\end{tabular}


Table 3 Postoperative metastatic patterns of patients with stage III colon cancer after curative treatment

\begin{tabular}{|c|c|c|c|}
\hline $\begin{array}{l}\text { Metastatic } \\
\text { parameters }\end{array}$ & $\begin{array}{l}\text { Right-sided colon } \\
(\mathrm{n}=110, \%)\end{array}$ & $\begin{array}{l}\text { Left-sided colon } \\
(n=164, \%)\end{array}$ & $P$-value \\
\hline \multicolumn{3}{|c|}{ Postoperative metastasis } & 0.396 \\
\hline Yes & $24(21.8)$ & $29(17.7)$ & \\
\hline No & $86(78.2)$ & $135(82.3)$ & \\
\hline $\begin{array}{l}\text { Liver } \\
\text { metastasis }\end{array}$ & & & 0.260 \\
\hline Yes & $6(5.5)$ & $15(9.1)$ & \\
\hline No & $104(94.5)$ & 149 (90.9) & \\
\hline \multicolumn{4}{|l|}{ metastasis } \\
\hline Yes & $5(2.7)$ & $9(5.5)$ & \\
\hline No & $107(97.3)$ & $155(94.5)$ & \\
\hline \multicolumn{3}{|c|}{ Abdominopelvic metastasis } & 0.003 \\
\hline Yes & $12(10.9)$ & $4(2.4)$ & \\
\hline No & $98(89.1)$ & $160(97.6)$ & \\
\hline
\end{tabular}

metastasis, and lung metastasis were not significantly different between RCC and LCC (all $P>0.050$ ).

The 3-year DFS and OS rates were $81.8 \%$ and $91.4 \%$ in the entire study population, respectively. Although no significant difference was observed in the 3-year DFS rate $(78.7 \%$ vs. $83.8 \% ; P=0.303$; Figure $2 \mathrm{~A}$ ), RCC patients exhibited a significantly worse 3 -year OS rate than LCC patients (86.1\% vs. $95.0 \% ; P=0.016$; Figure $2 \mathrm{~B}$ ). There were no differences in DFS and OS rates among patients with stage IIIA-B colon cancer between RCC and LCC (DFS: $86.7 \%$ vs. $87.2 \%$; $P=0.944$; Figure $2 \mathrm{C}$; OS: $91.6 \%$ vs. $96.0 \%$; $P=0.526$; Figure 2D). However, among patients with stage IIIC colon cancer, the 3-year DFS rate was slightly lower, and OS rates were significantly lower in RCC patients than in LCC patients (DFS: $52.2 \%$ vs. $72.2 \%, P=0.062$, Figure $2 \mathrm{E}$; OS: $63.7 \%$ vs. $91.5 \%, P=0.003$, Figure $2 \mathrm{~F}$ ). As shown in Table 4, univariate analysis revealed that RCC (HR: 2.243; 95\% CI: $1.089-4.619 ; P=0.020)$ was a significant negative predictor of 3-year OS, whereas 6-8 cycles of adjuvant chemotherapy (HR: 0.308; 95\% CI: 0.148-0.640; $P=0.002$ ) were a significant protective factor for the 3-year OS. In multivariate analysis, tumor location (HR: 2.213; 95\% CI: $1.063-4.606 ; P=0.002$ ) and cycles of adjuvant chemotherapy (HR: 0.325; 95\% CI: 0.156-0.678; $P=0.003$ ) were identified as independent predictors of 3-year OS.

The association between long-term survival and different total cycles of adjuvant chemotherapy was evaluated in LCC and RCC patients. Among patients with LCC, no significant difference was observed for 3-year DFS and OS rates between those who received 6-8 cycles and those who received less than 6 cycles of adjuvant chemotherapy (DFS: $82.3 \%$ vs.
92.0\%, $P=0.418$, Figure 3A; OS: $95.5 \%$ vs. $92.0 \% P=$ 0.353 , Figure $3 \mathrm{~B})$. However, among RCC patients, the 3 -year DFS and OS rates were significantly higher for patients who received 6-8 cycles of adjuvant chemotherapy than for those who received less than 6 cycles of adjuvant chemotherapy (DFS: $85.2 \%$ vs. $58.5 \%, P=0.003$, Figure 3C; OS: $91.4 \%$ vs. $69.8 \%, P=0.002$, Figure $3 \mathrm{D})$.

\section{Discussion}

This study demonstrated a significant difference in prognostic outcome between patients with right- and left-sided stage III colon cancers who had undergone curative resection and XELOX adjuvant chemotherapy. Patients with RCC exhibited a worse 3-year OS than those with LCC. Additionally, the difference was larger in patients with stage IIIC colon cancer. Furthermore, we found that patients with RCC treated with 6-8 cycles of XELOX adjuvant chemotherapy exhibited longer survival, but patients with LCC did not. Importantly, tumor location was demonstrated to be an independent prognostic factor for 3-year OS in this study.

Previous studies have also reported that patients with stage III RCC likely present a significantly increased mortality risk compared with those with LCC. ${ }^{17,21,22}$ By analyzing various stages of colorectal cancer, Huang et al observed a poorer OS in patients with RCC than those with LCC, whereas significant differences were only noted in stage III patients. ${ }^{21}$ For patients treated with curative resection and subsequent oxaliplatin-based adjuvant chemotherapy, Sinicrope et al found that RCC was significantly associated with a shorter DFS than LCC in patients with BRAF-wild-type stage III colon cancer. ${ }^{17}$ Similarly, Zhang et al demonstrated that both the 5-year recurrence-free survival and 5-year OS were significantly different and showed that survival gradually decreased for tumors from the cecum to the sigmoid colon. ${ }^{22}$ However, how many adjuvant chemotherapy cycles were administered was not discussed in the abovementioned studies. It was noted that a shorter duration of adjuvant chemotherapy generated worse prognostic outcomes in patients with colon cancer. ${ }^{23}$ In our study, we identified 6-8 cycles of adjuvant chemotherapy as a protective factor for 3-year OS, whereas chemotherapy cycles were not significantly different between colon cancers, and at least 6 cycles (median) were received by patients harboring tumors at each site. Therefore, the different efficacy of oxaliplatin adjuvant chemotherapy depending on colon cancer location might be an important factor affecting survival.

Here, the different clinical and biological profiles of RCC and LCC might contribute to different prognostic benefits 
A

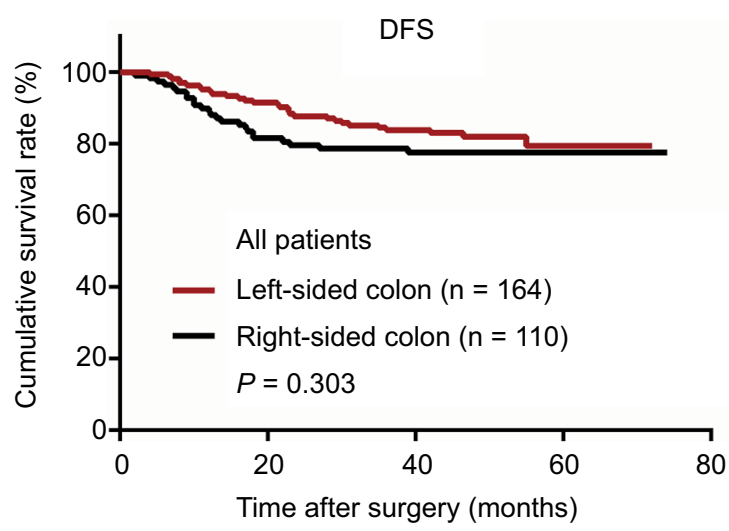

C

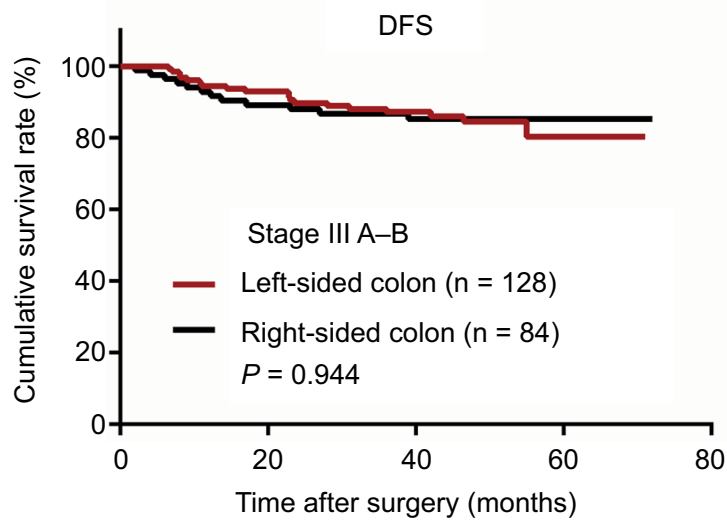

E

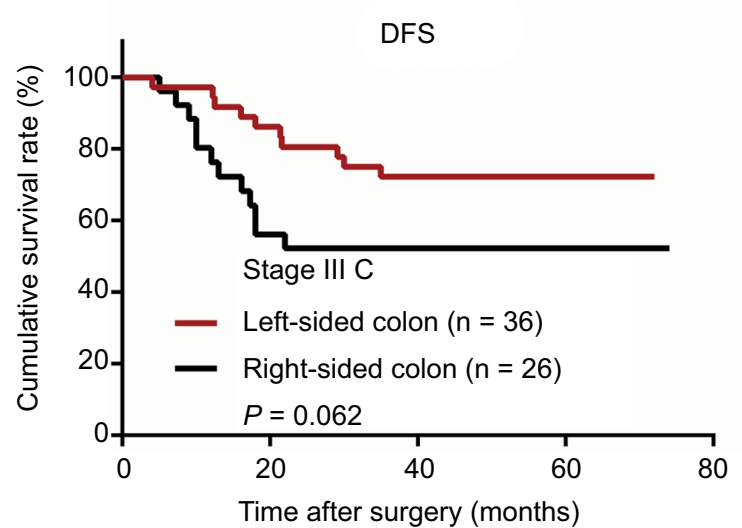

B

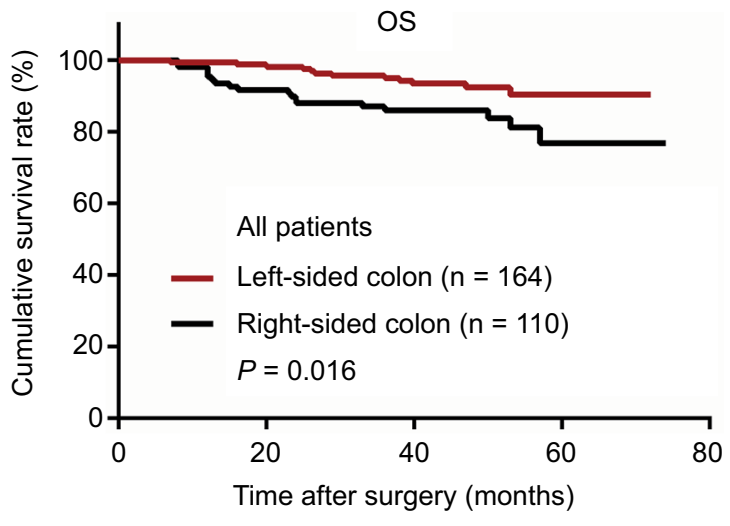

D

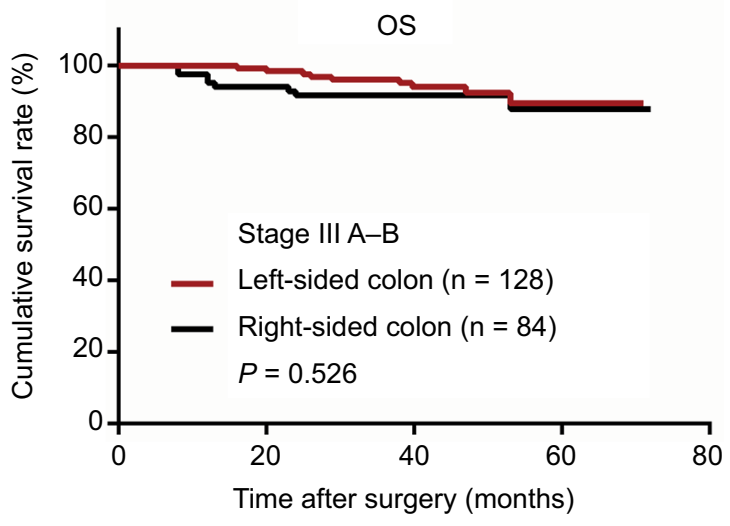

$\mathbf{F}$

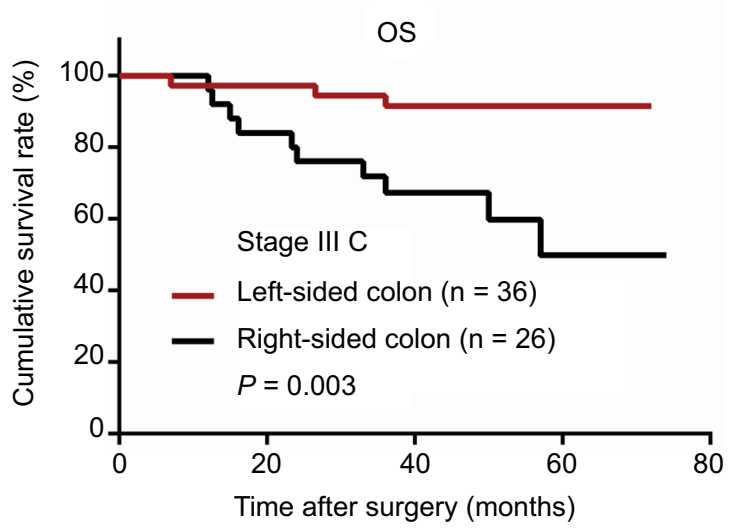

Figure 2 Kaplan-Meier curves of patients with stage III colon cancer grouped by right- and left-sided colon and stratified by stage.

Notes: (A) Disease-free survival (DFS) of all patients. (B) Overall survival (OS) of all patients. (C) DFS of patients with stage IIIA-B colon cancer. (D) OS of patients with stage IIIA-B colon cancer. (E) DFS of patients with stage IIIC colon cancer. (F) OS of patients with stage IIIC colon cancer.

from oxaliplatin-based adjuvant chemotherapy. Generally, RCC symptoms are easily ignored or often nonspecific. Thus, patients were more likely to have experienced a long period of tumor progression at the time of diagnosis and treatment. We found that patients with RCC more commonly had severe anemia (hemoglobin $<90 \mathrm{~g} / \mathrm{L}$ ) and a larger tumor $(>4 \mathrm{~cm}$ ) at the time of surgery. Previous studies have also observed that advanced pathologic $\mathrm{T}(\mathrm{pT})$ stage, poor nutritional status (weakness and weight loss), and older age occurred more frequently with RCC. ${ }^{13,24}$ A higher tumor burden and severe nutritional deficiency might lead to poor survival in RCC patients. In addition, the diverse pathological features 
Table 4 Univariate and multivariate analyses of prognostic factors for overall survival in patients with stage III colon cancer who received curative treatment

\begin{tabular}{|c|c|c|c|c|}
\hline \multirow[t]{2}{*}{ Variables } & \multicolumn{2}{|l|}{ Univariate } & \multicolumn{2}{|l|}{ Multivariate } \\
\hline & HR (95\% Cl) & $P$-value & HR $(95 \% \mathrm{Cl})$ & $P$-value \\
\hline Age, years (>60 vs. $\leq 60)$ & $1.390(0.66 \mathrm{I}-2.925)$ & 0.385 & & \\
\hline Gender (male vs. female) & $1.902(0.87 I-4.154)$ & 0.107 & & \\
\hline Baseline BMI, $\mathrm{kg} / \mathrm{m}^{2}(<18.5$ vs. $\geq 18.5)$ & $1.892(0.724-4.944)$ & 0.193 & & \\
\hline Baseline hemoglobin, $g / L(<90$ vs. $\geq 90)$ & $0.818(0.285-2.346)$ & 0.709 & & \\
\hline Tumor size, $\mathrm{cm}(>4$ vs. $\leq 4)$ & $1.046(0.5 \mathrm{II}-2.140)$ & 0.902 & & \\
\hline Differentiation (poor vs. well/moderate) & I.I8I (0.525-2.656) & 0.687 & & \\
\hline T stage (T4 vs. TI-3) & $1.709(0.797-3.668)$ & 0.169 & & \\
\hline Number of resected lymph nodes $(<12$ vs. $\geq 12)$ & $1.080(0.480-2.429)$ & 0.853 & & \\
\hline $\mathrm{N}$ stage (N2 vs. NI) & $1.487(0.716-3.089)$ & 0.287 & & \\
\hline Preoperative serum CEA, ng/mL ( $>5$ vs. $\leq 5)$ & $0.987(0.479-2.034)$ & 0.972 & & \\
\hline Adjuvant chemotherapy, cycle (6-8 vs. <6) & $0.308(0.148-0.640)$ & 0.002 & $0.329(0.158-0.685)$ & 0.003 \\
\hline Tumor location (right-sided colon vs. left-sided colon) & $2.243(1.089-4.619)$ & 0.020 & $2.213(1.063-4.606)$ & 0.002 \\
\hline
\end{tabular}

Abbreviations: BMI, body mass index; HR, hazard ratio; CEA, carcinoembryonic antigen.

A

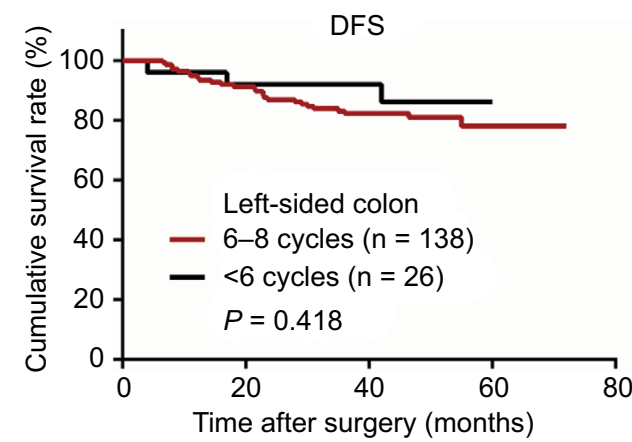

C

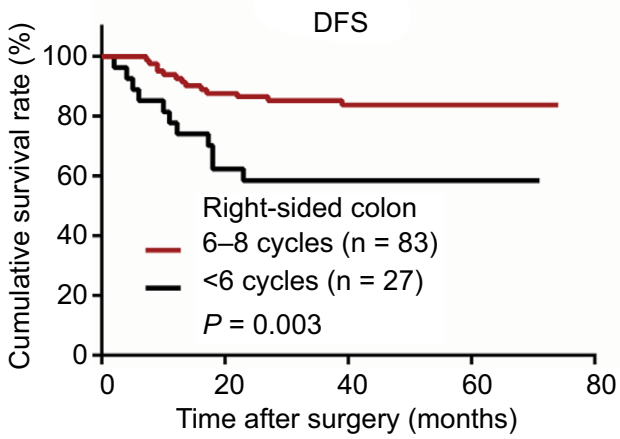

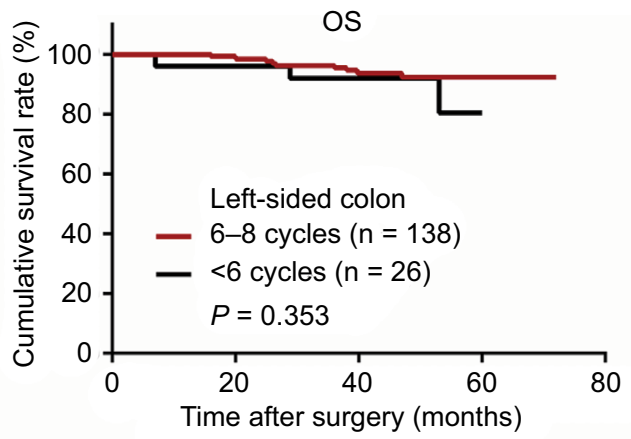

D

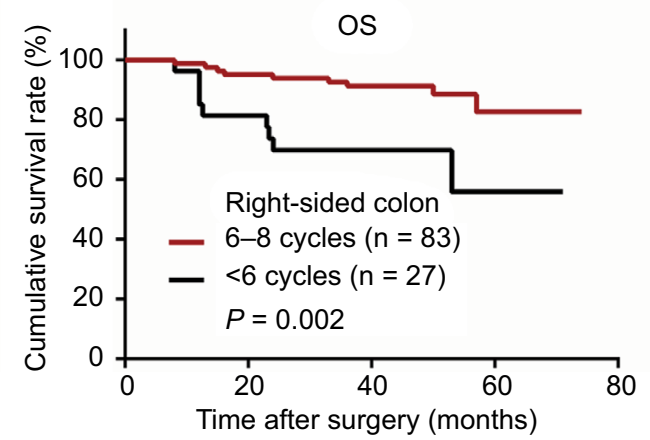

Figure 3 Kaplan-Meier curves of patients with stage III colon cancer in right- and left-sided colon stratified by cycles of XELOX* adjuvant chemotherapy.

Notes: (A) Disease-free survival (DFS) in left-sided colon cancer. (B) Overall survival (OS) in left-sided colon cancer. (C) DFS in right-sided colon cancer. (D) OS in right-sided colon cancer. *XELOX adjuvant chemotherapy was administered as follows: oxaliplatin at a dose of $130 \mathrm{mg} / \mathrm{m}^{2}$ was administered intravenously on day I, and capecitabine was administered orally at a dosage of $1000 \mathrm{mg} / \mathrm{m}^{2}$ twice daily on days $1-14$ for a 3-week cycle.

and molecular biology of site-specific colon cancers might account for the different prognoses. Poor differentiation, increased frequency of vascular invasion, and an invasive tumor border are commonly observed in RCC. ${ }^{25,26}$ In fact, these aggressive and advanced tumor features likely contribute to postoperative metastasis. This study found that the incidence of abdominopelvic metastasis was significantly higher in RCC patients than in LCC patients, which has been demonstrated as a risk factor resulting in the worst survival outcome. ${ }^{27}$ The levels of specific biomarkers, including MSI, CIMP level, and BRAF and KRAS mutations, gradually increase from the distal to the proximal colon. ${ }^{14,28}$ BRAF 
V600E mutation and KRAS exon 2 mutations are correlated with a worse prognosis in stage III colon cancers. ${ }^{18,29}$ Herein, these biological distinctions between RCC and LCC could also be translated into important differences in survival.

To date, a general consensus has been developed that tumor location is an important variable in the management of colon cancer. ${ }^{30,31}$ Our study found a different survival impact for adjuvant chemotherapy duration in patients with either RCC or LCC. A survival benefit from the full course of adjuvant chemotherapy was only observed in patients with RCC. This finding indicated that a full course of adjuvant chemotherapy is not equally effective to treat different sided colon cancers. However, the underlying mechanisms creating different outcomes after a full course of adjuvant chemotherapy for RCC and LCC remain unclear. We considered that advanced tumors were more frequently observed on the right side, and these tumors might benefit from more intensive chemotherapy. ${ }^{5,32}$ In this study, $75.5 \%$ of patients with RCC successfully completed 6-8 cycles of the XELOX chemotherapy regimen with good tolerability and comparable toxicities to LCC. Therefore, we recommend 6-8 cycles of XELOX chemotherapy for patients with RCC to minimize the risk of postoperative tumor recurrence.

\section{Limitations}

First, this retrospective study included an uncontrolled methodology and a limited number of patients from a single cohort. Therefore, the findings need to be validated in a larger prospective study. Second, the short follow-up time duration was insufficient to evaluate 5-year survival outcomes. This limitation may have led to underestimation of the prognostic impact of tumor location on DFS. Moreover, tumor molecular features, such as MSI, CIMP level, and BRAF and KRAS mutations, were not evaluated in this study; this analysis would further enhance our understanding of the prognostic impact of molecular markers on site-specific colon cancers. Nevertheless, our findings suggest that tumor location is an important factor that should guide adjuvant chemotherapy in stage III colon cancer.

\section{Conclusion}

This study demonstrated that tumors arising on different sides generate different prognostic outcomes in patients with stage III colon cancer who received adjuvant chemotherapy after curative resection. The full course of adjuvant chemotherapy might only benefit RCC patients. The tumor location is suggested to be an important clinical factor that can optimize the therapeutic adjuvant chemotherapy strategies. Further prospective studies are warranted to confirm these findings.

\section{Acknowledgments}

The authors deeply appreciate the help from all colleagues of the Department of Colorectal Surgery at Sun Yat-sen University Cancer Center who were involved in performing the treatment for current study. The authenticity of this article has been validated by uploading the key raw data onto the Research Data Deposit public platform (www.researchdata. org.cn), with the approval number as RDDA2017000388. This work was funded by grants from National Natural Science Foundation of China (No. 81772595 and No. 81502459), the Sun Yat-sen University Clinical Research 5010 Program (No.2015024 and No. 2013013), and the Science and Technology Project in Guangdong Province (No. 2013B021800146).

\section{Disclosure}

The authors report no conflicts of interest in this work.

\section{References}

1. Benson AB 3rd, Venook AP, Bekaii-Saab T, et al; National Comprehensive Cancer Network. Colon cancer, version 3.2014. J Natl Compr Canc Netw. 2014;12(7):1028-1059.

2. Labianca R, Nordlinger B, Beretta GD, et al; ESMO Guidelines Working Group. Early colon cancer: ESMO Clinical Practice Guidelines for diagnosis, treatment and follow-up. Ann Oncol. 2013;24 Suppl 6:vi64-vi72.

3. Haller DG, Tabernero J, Maroun J, et al. Capecitabine plus oxaliplatin compared with fluorouracil and folinic acid as adjuvant therapy for stage III colon cancer. J Clin Oncol. 2011;29(11):1465-1471.

4. Yothers G, O'Connell MJ, Allegra CJ, et al. Oxaliplatin as adjuvant therapy for colon cancer: updated results of NSABP C-07 trial, including survival and subset analyses. J Clin Oncol. 2011;29(28):3768-3774.

5. André $T$, Boni $C$, Navarro $M$, et al. Improved overall survival with oxaliplatin, fluorouracil, and leucovorin as adjuvant treatment in stage II or III colon cancer in the MOSAIC trial. J Clin Oncol. 2009;27(19):3109-3116.

6. Haller DG, Catalano PJ, Macdonald JS, et al. Phase III study of fluorouracil, leucovorin, and levamisole in high-risk stage II and III colon cancer: final report of Intergroup 0089. J Clin Oncol. 2005;23(34):8671-8678.

7. Murphy CC, Harlan LC, Warren JL, Geiger AM. Race and insurance differences in the receipt of adjuvant chemotherapy among patients with stage III colon cancer. J Clin Oncol. 2015;33(23):2530-2536.

8. Stotz M, Pichler M, Absenger G, et al. The preoperative lymphocyte to monocyte ratio predicts clinical outcome in patients with stage III colon cancer. Br J Cancer. 2014;110(2):435-440.

9. van Erning FN, Creemers GJ, De Hingh IH, Loosveld OJ, Goey SH, Lemmens VE. Reduced risk of distant recurrence after adjuvant chemotherapy in patients with stage III colon cancer aged 75 years or older. Ann Oncol. 2013;24(11):2839-2844.

10. Bufill JA. Colorectal cancer: evidence for distinct genetic categories based on proximal or distal tumor location. Ann Intern Med. 1990;113(10):779-788.

11. Yamauchi M, Lochhead P, Morikawa T, et al. Colorectal cancer: a tale of two sides or a continuum? Gut. 2012;61(6):794-797.

12. Siegel RL, Miller KD, Fedewa SA, et al. Colorectal cancer statistics, 2017. CA Cancer J Clin. 2017;67(3):177-193. 
13. Benedix F, Kube R, Meyer F, Schmidt U, Gastinger I, Lippert H; Colon/ Rectum Carcinomas (Primary Tumor) Study Group. Comparison of 17,641 patients with right- and left-sided colon cancer: differences in epidemiology, perioperative course, histology, and survival. Dis Colon Rectum. 2010;53(1):57-64.

14. Yamauchi M, Morikawa T, Kuchiba A, et al. Assessment of colorectal cancer molecular features along bowel subsites challenges the conception of distinct dichotomy of proximal versus distal colorectum. Gut. 2012;61(6):847-854.

15. Suttie SA, Shaikh I, Mullen R, Amin AI, Daniel T, Yalamarthi S. Outcome of right- and left-sided colonic and rectal cancer following surgical resection. Colorectal Dis. 2011;13(8):884-889.

16. Yahagi M, Okabayashi K, Hasegawa H, Tsuruta M, Kitagawa Y. The worse prognosis of right-sided compared with left-sided colon cancers: a systematic review and meta-analysis. J Gastrointest Surg. 2016;20(3):648-655.

17. Sinicrope FA, Mahoney MR, Smyrk TC, et al. Prognostic impact of deficient DNA mismatch repair in patients with stage III colon cancer from a randomized trial of FOLFOX-based adjuvant chemotherapy. $J$ Clin Oncol. 2013;31(29):3664-3672.

18. Blons H, Emile JF, Le Malicot K, et al; PETACC-8 Study Investigators. Prognostic value of KRAS mutations in stage III colon cancer: post hoc analysis of the PETACC8 phase III trial dataset. Ann Oncol. 2014;25(12):2378-2385.

19. Wang F, Bai L, Liu TS, et al. Right-sided colon cancer and left-sided colorectal cancers respond differently to cetuximab. Chin J Cancer. 2015;34(9):384-393.

20. Weiss J, Schumacher J, Allen GO, et al. Adjuvant chemotherapy for stage II right-sided and left-sided colon cancer: analysis of SEER-medicare data. Ann Surg Oncol. 2014;21(6):1781-1791.

21. Huang CW, Tsai HL, Huang MY, et al. Different clinicopathologic features and favorable outcomes of patients with stage III left-sided colon cancer. World J Surg Oncol. 2015;13:257.

22. Zhang Y, Ma J, Zhang S, et al. A prognostic analysis of 895 cases of stage III colon cancer in different colon subsites. Int J Colorectal Dis. 2015;30(9):1173-1183.
23. Chau I, Norman AR, Cunningham D, et al. A randomised comparison between 6 months of bolus fluorouracil/leucovorin and 12 weeks of protracted venous infusion fluorouracil as adjuvant treatment in colorectal cancer. Ann Oncol. 2005;16(4):549-557.

24. Nawa T, Kato J, Kawamoto H, et al. Differences between right- and left-sided colon cancer in patient characteristics, cancer morphology and histology. J Gastroenterol Hepatol. 2008;23(3):418-423.

25. Tentes AA, Korakianitis OS, Kakolyris S, et al. Differences between right- and left-sided colon carcinomas. J BUON. 2010;15(2):285-289.

26. Snaebjornsson P, Jonasson L, Jonsson T, Möller PH, Theodors A, Jonasson JG. Colon cancer in Iceland--a nationwide comparative study on various pathology parameters with respect to right and left tumor location and patients age. Int J Cancer. 2010;127(11):2645-2653.

27. Franko J, Shi Q, Meyers JP, et al; Analysis and Research in Cancers of the Digestive System (ARCAD) Group. Prognosis of patients with peritoneal metastatic colorectal cancer given systemic therapy: an analysis of individual patient data from prospective randomised trials from the Analysis and Research in Cancers of the Digestive System (ARCAD) database. Lancet Oncol. 2016;17(12):1709-1719.

28. Phipps AI, Limburg PJ, Baron JA, et al. Association between molecular subtypes of colorectal cancer and patient survival. Gastroenterology. 2015;148(1):77-87.e2.

29. Sinicrope FA, Shi Q, Smyrk TC, et al. Molecular markers identify subtypes of stage III colon cancer associated with patient outcomes. Gastroenterology. 2015;148(1):88-99.

30. Shen H, Yang J, Huang Q, et al. Different treatment strategies and molecular features between right-sided and left-sided colon cancers. World J Gastroenterol. 2015;21(21):6470-6478.

31. Lee GH, Malietzis G, Askari A, Bernardo D, Al-Hassi HO, Clark SK. Is right-sided colon cancer different to left-sided colorectal cancer? - a systematic review. Eur J Surg Oncol. 2015;41(3):300-308.

32. Shah MA, Renfro LA, Allegra CJ, et al. Impact of patient factors on recurrence risk and time dependency of oxaliplatin benefit in patients with colon cancer: analysis from modern-era adjuvant studies in the adjuvant colon cancer end points (ACCENT) database. J Clin Oncol. 2016;34(8):843-853.
Cancer Management and Research

\section{Publish your work in this journal}

Cancer Management and Research is an international, peer-reviewed open access journal focusing on cancer research and the optimal use of preventative and integrated treatment interventions to achieve improved outcomes, enhanced survival and quality of life for the cancer patient. The manuscript management system is completely online and includes

\section{Dovepress}

a very quick and fair peer-review system, which is all easy to use. Visit http://www.dovepress.com/testimonials.php to read real quotes from published authors. 